\title{
Incidence and causes of neonatal hyperbilirubinemia in a center of Catania
}

\author{
Marco Sciuto' \\ Gaetano Bertino² \\ Mariangela Zocco ${ }^{3}$ \\ Ignazio Vecchio ${ }^{4}$ \\ Rocco Raffaele ${ }^{4}$ \\ Rosario R Trifiletti ${ }^{5}$ \\ Piero Pavone 3,6 \\ 'Neonatal Care Section, Valsalva \\ Hospital, Catania, Italy; ${ }^{2}$ Department \\ of Internal Medicine, Hospital S Marta \\ University of Catania, Italy; \\ ${ }^{3}$ Pediatric Unit, Department \\ of Pediatric Hospital Civile, \\ Ragusa, Italy; ${ }^{4}$ Department \\ of Neurology, University of Catania, \\ Italy; ${ }^{5}$ Department of Neurology, \\ Morristown Memorial Hospital,

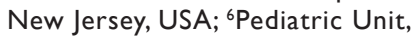 \\ Department of Pediatric and Pediatric \\ Neurology, University of Catania, Italy
}

Correspondence: Piero Pavone Divisione di Clinica Pediatrica, Clinica Pediatrica, Università di Catania, Via Santa Sofia 78 - 95I25 Catania, Italy

Tel +39095 $3782478 / 3782394$

Fax +39095222532

Email ppavone@mbox.unict.it

\begin{abstract}
Aim and scope: We conducted this study to estimate the incidence of hyperbilirubinemia in a small neonatal care unit in Catania, Italy, and to determine the underlying causes, which would be of value in identifying and implementing strategies to prevent morbidity from this condition.
\end{abstract}

Background: Management of hyperbilirubinemia remains a challenge for neonatal medicine because of the risk for serious neurological complications related to the toxicity of bilirubin.

Methods: From January 2006 to January 2007, we screened 525 newborns born at the Neonatal Care Unit of Valsalva Hospital in Catania, Italy. Infants aged 3-5 days and with unconjugated hyperbilirubinemia were included for assessment if they had a peak serum total bilirubin level exceeding $6 \mathrm{mg} / \mathrm{dl}(102 \mu \mathrm{mol} / \mathrm{L})$. Sex, birth weight, gestational age, breast feeding, type of birth, presence of facial bruising (including cephalohematoma) and ABO group were noted. Patients with Toxoplasma or Cytomegalovirus infection, hepatic insufficiency, or suspected drug-induced hyperbilirubinemia were excluded from more detailed analysis.

Results: Our year-long nursery sample examined otherwise healthy-appearing term infants for the prevalence of hyperbilirubinemia (defined as bilirubin levels exceeding $6 \mathrm{mg} / \mathrm{dL}[11 \mathrm{~mol} / \mathrm{L}]$ ). We found hyperbilirubinemia in 19\% (100/525). Among the patients with hyperbilirubinemia, almost all (99\%) had peak levels of bilirubin $<20 \mathrm{mg} / \mathrm{dL}$, levels which are generally considered to be potentially neurotoxic.

Conclusions: In our clinic experience, hyperbilirubinemia was generally a serious medical issue and one whose etiology can usually be well defined.

Keywords: hyperbilirubinemia, newborns, incidence, breastfeeding

\section{Introduction}

Severe hyperbilirubinemia continues to be the most common cause of neonatal readmission for hospitals in North America. ${ }^{1-5}$ This pattern continues despite attempts to identify newborns at risk of clinically important hyperbilirubinemia before they are discharged from hospital. ${ }^{6-9}$ Long-term results of severe hyperbilirubinemia, including bilirubin encephalopathy and kernicterus, were thought to be rare since the advent of exchange transfusion, maternal Rh immunoglobulin prophylaxis, and phototherapy. ${ }^{10-12}$ However, cases of kernicterus have been reported recently in healthy near-term and term infants without evidence of hemolytic disease or other risk factors. ${ }^{13,14}$

We begin our study of this problem with a survey of a single clinic experience over a year with respective to severity and probable causes of hyperbilirubinemia.

\section{Background}

Management of hyperbilirubinemia remains a challenge for neonatal medicine because of the risk for serious neurological complications related to the toxicity of bilirubin. The neonatal hyperbilirubinemia practice guidelines published in 2004 by the American Academy of Pediatrics (AAP) expresses the pediatric community's concern regarding bilirubin-induced neurological pathology. Risk factors recognized 
to be associated with severe hyperbilirubinemia in newborns have included jaundice in the first 24 hours of life, jaundice noted before discharge from hospital, a sibling who had jaundice treated with phototherapy, near-term gestational age of 35-36 weeks, and the presence of infant with bruising or cephalohematoma.

\section{Materials and methods}

From January 2006 to January 2007, we screened 525 newborns born at the Neonatal Care Unit of Valsalva Hospital in Catania, Italy. Infants aged 3-5 days and with unconjugated hyperbilirubinemia were included for assessment if they had a peak serum total bilirubin level exceeding $6 \mathrm{mg} / \mathrm{dl}(102 \mu \mathrm{mol} / \mathrm{L})$. Sex, birth weight, gestational age, breast feeding, type of birth, presence of facial bruising (including cephalohematoma), and ABO group were noted. Patients with Toxoplasma or Cytomegalovirus infection, hepatic insufficiency, or suspected drug-induced hyperbilirubinemia were excluded from more detailed analysis.

We focused on healthy term infants without risk factors. Infants who had known Rh iso-immunization were excluded since antenatal and postnatal strategies already exist to prevent the occurrence of severe neonatal hyperbilirubinemia from this cause. Infants who were born at less than 36 weeks' gestational age were also excluded as well as newborn with severe facial bruising or cephalohematoma from more detailed analysis.

Data were summarized using descriptive statistics. Continuous variables were analyzed using the independent Student's t-test and Fisher's exact test. A chi square test was used to test associations between all other categorical variables.

\section{Results}

We found 100 children with unconjugated hyperbilirubinemia. Causes identified by laboratory investigations include $\mathrm{Rh}$ and $\mathrm{ABO}$ incompatibility, as well as glucose-6-phosphate dehydrogenase (G6PD) deficiency. We found potentially neurotoxic levels of hyperbilirubinema in only one case, with value of peak bilirubin of $20 \mathrm{mg} / \mathrm{dL}$ (342 mmol/L), treated with phototherapy. The other 99 cases had milder hyperbilirubinemia and did not receive any treatment.

In our series, we found breastfeeding in 65 cases, cesarean section in 61, and mild facial bruising in 16. Medicaments during the pregnancy were used in 39 cases and post-pregnancy in 35 (Table 1). A single case showed evidence of liver disease (Gilbert disease). Cytomegalovirus and toxoplasmosis infection were found in $2 \%$ and $6 \%$ of the cases, respectively (Table 1). Maternal microcytosis was found in two cases.
Table I Pregnancy factors

\begin{tabular}{llll}
\hline & Yes & No & Total \\
\hline Breastfeeding & 65 & 35 & 100 \\
Cesarian section & 61 & 39 & 100 \\
Spontaneous delivery & 39 & 61 & 100 \\
Bruising face & 16 & 84 & 100 \\
Drugs in pregnancy & 39 & 61 & 100 \\
Drugs after pregnancy & 35 & 65 & 100 \\
Liver disease & 1 & 99 & 100 \\
Cytomegalovirus in pregnancy & 2 & 98 & 100 \\
Microcytemic mother & 2 & 98 & 100 \\
Toxoplasmosis in pregnancy & 6 & 94 & 100 \\
\hline
\end{tabular}

We also evaluated the ABO group in the mothers and in the newborns (Table 2). No significant differences were found in the examined group. No other risk factors were found in those patients.

We tried to analyze the characteristics of neonatal jaundice. We took in consideration over 525 children, 100 infants with a value of bilirubin level of more than $6 \mathrm{mg} / \mathrm{dl}(102 \mu \mathrm{mol} / \mathrm{L})$ (Table 3$)$.

\section{Discussion}

Hyperbilirubinemia is felt to be a benign condition for infants born at term or near-term gestation. In around 5\% of healthy term infants, however, serum bilirubin values exceed $17 \mathrm{mg} / \mathrm{dL}$ (291 mmol/L), a value which the AAP deems significant. ${ }^{4}$ Levels exceeding $20 \mathrm{mg} / \mathrm{dL}$ (342 mmol/L) occur in $1.2 \%$ of healthy newborn infants. ${ }^{11}$ The vast majority of infants with serum bilirubin values of $20 \mathrm{mg} / \mathrm{dL}$ remain well; mostly need minimal care other the occasional use of phototherapy and careful monitoring of serum levels. Before 1990, kernicterus in the previously healthy-term infant was extraordinarily rare and for most pediatricians, it was a

Table 2 Blood groups

\begin{tabular}{lll}
\hline Blood group & Maternal group & Children group \\
\hline $0-$ & 5 & 6 \\
$0+$ & 52 & 48 \\
$\mathrm{~A}-$ & 2 & 2 \\
$\mathrm{~A}+$ & 17 & 29 \\
$\mathrm{~B}-$ & 6 & 1 \\
$\mathrm{~B}+$ & 16 & 13 \\
$\mathrm{AB}+$ & 2 & 1 \\
$\mathrm{AB}-$ & 0 & 0 \\
Total & 100 & 100 \\
\hline
\end{tabular}


Table 3 Characteristics

\begin{tabular}{l} 
Characteristics \\
\hline No $(\%)$ of infants* \\
$\mathrm{n}=100$ \\
Gestational age: wk, mean (SD) $38.5(\mathrm{I} .4)$ \\
Sex: male 42 and female 68 \\
Birth weight, g, mean (SD) $3250(+/-489)$ \\
Age at presentation of jaundice, h, mean (SD) 78.5 \\
Breast-feeding 65 \\
Peak total bilirubin level, $\propto$ mol/L, \\
mean (SD) \\
I86 $( \pm 76)$
\end{tabular}

disease they were unlikely to see in their practice lifetimes. Since 1990, there has been an increase in the number of reported cases of kernicterus in the United States. ${ }^{7}$ Thirty-one cases have been reported in term infants who were well at the time of hospital discharge, and several additional cases have occurred in near-term infants. Although it is unknown whether there is an actual increase in the incidence of kernicterus in the United States or simply better detection, reported cases are thought to be attributable to a variety of events including: shortened hospital stays with inconsistent follow-up beyond discharge; an increase in the frequency of breastfeeding; and a lack of concern about high bilirubin levels among pediatric care providers.

Although the relationship between hyperbilirubinemia and brain injury in healthy term infants has been recently questioned, ${ }^{17}$ in a reevaluation of the data from the Collaborative Perinatal Project, Newman and Klebanoff ${ }^{10}$ could not demonstrate a relationship between bilirubin levels $26 \mathrm{mg} / \mathrm{dL}$ $(342 \mathrm{uM} / \mathrm{L})$ and an abnormal neurologic examination.

Breastfeeding is still debated as probably an important variable in these patients. In the present series, almost $65 \%$ of hyperbilirubemic patients were breastfed, as it has been for the other cases of kernicterus reported in the literature. ${ }^{3,7,17}$ In our series, no significance statistical difference in bilirubin levels were found according to whether or not the infant was breastfed.

In a case-controlled study Maisels ${ }^{8}$ found that hyperbilirubinemia was the major reason for hospital readmission during the first two weeks of life. Moreover, the majority of the jaundiced infants in their study were breastfed and had a greater mean weight loss since birth than nonjaundiced controls, suggesting that even mild degrees of dehydration in conjunction with breastfeeding impacts on the severity of hyperbilirubinemia.
Our series comes from a small center in Catania, Italy. Catania is one of the sunniest spots on Earth, with approximately 225 sunny days per year (which does not include partly cloudy days). It is possible that this plays some role in the relatively low incidence of hyperbilirubinemia in our studies as compared to previous studies. Furthermore future studies should investigate the presence of individual susceptibility, environmental factors, especially environmental climate (daylight), and genetic factors to explain the different incidences of the disease, and the frequency of such findings in infants with moderate degrees of hyperbilirubinemia and possible modes of prevention and new therapeutic strategies.

\section{Disclosure}

The authors report no conflicts of interest in this work. We are grateful to Prof. Lorenzo Pavone for helpful suggestions and critical review of the manuscript. We wish to thank Prof A Bridgewood and International Science Editing, Compuscript Ltd., Shannon Industrial Estate West Shannon, Co., Clare, Republic of Ireland, for editing the manuscript.

\section{References}

1. Liu LL, Clemens CJ, Shay DK, Davis RL, Novack AH. The safety of newborn early discharge: the Washington State experience. JAMA. 1997;278:293-298.

2. Maisels MJ, Newman TB. Jaundice in full-term and near-term babies who leave the hospital within 36 hours. Clin Perinatol. 1998;25:295-302.

3. Johnson L, Bhutani VK. Guidelines for management of the jaundiced term and near-term Infant. Clin Perinatol. 1998;25:555-574.

4. American Academy of Pediatrics, Provisional Committee for Quality Improvement and Subcommittee on Hyperbilirubinemia. Practice parameter: management of hyperbilirubinemia in the healthy term newborn. Pediatrics. 1994;94:558-565.

5. Dennery PA, Rhine WD, Stevenson DK. Neonatal jaundice-what now? Clin Pediatr. 1995;34:103-107.

6. Newman TB, Maisels MJ. Evaluation and treatment of jaundice in the term newborn: a kinder and gentler approach. Pediatrics. 1992; 89: 809-818.

7. Brown AK, Johnson L. Loss of concern about jaundice and the reemergence of kernicterus in full-term infants in the era of managed care. In: Faranoff AA, Klaus MH, editors. The Year Book of Neonatal and Perinatal Medicine. St Louis, MO: Mosby-Year Book; 1996. p. 17-28.

8. Maisels MJ, Kring E. Length of stay, jaundice, and hospital readmission. Pediatrics. 1998;101:995-998.

9. Volpe JJ. Neurology of the Newborn. Philadelphia, PA: WB Saunders; 1995:490-514.

10. Newman TB, Klebanoff MA. Neonatal hyperbilirubinemia and longterm outcome: another look at the Collaborative Perinatal Project. Pediatrics. 1993;92:651-657.

11. Maisels MJ, Gifford K, Antle CE, et al. Normal serum bilirubin levels in the newborn and the effect of breast feeding. Pediatrics. 1986; $78: 837-843$.

12. Maisels MJ, Newman TB. Kernicterus in otherwise healthy, breastfed term newborns. Pediatrics. 1995;96:730-733.

13. Worley G, Erwin CW, Goldstein RF, et al. Delayed development of sensorineural hearing loss after neonatal hyperbilirubinemia: a case report with brain magnetic resonance imaging. Dev Med Child Neurol. 1996;38:271-278. 
14. Palmer C, Smith MB. Assessing the risk of kernicterus using nuclear magnetic resonance. Clin Perinatol. 1990;17:307-329.

15. Nakamura H, Satoshi T, Shimabuku R. Auditory and brainstem responses in newborn infants with hyperbilirubinemia. Pediatrics. 1985;73:703-708.
16. Gourley GR. Bilirubin metabolism and kernicterus. Adv Pediatr. 1997;44:173-229.

17. Newman TB, Maisels MJ. Does hyperbilirubinemia damage the brain of full-term infants? Clin Perinatol. 1990;17:331-358. 